\title{
Vivencias, expectativas y demandas de cuidadoras informales de pacientes en procesos de enfermedad de larga duración
}

\author{
Eugenia Gill García ${ }^{\mathrm{a}}$, María Escudero Carretero ${ }^{\mathrm{b}}$, M. Ángeles Prieto Rodríguez ${ }^{\mathrm{y}}$ y Antonio Frías Osuna ${ }^{d}$ \\ ${ }^{a}$ Diplomada en Enfermería. Licenciada en Sociología. Escuela Universitaria de Enfermería Universidad de Jaén. Jaén. España.

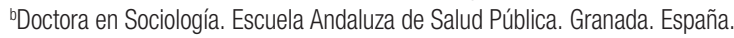 \\ 'Diplomada en Enfermería. Licenciada en Sociología. Escuela Andaluza de Salud Pública. Granada. España. \\ ¿Diplomado en Enfermería. Licenciado en Antropología. Escuela Universitaria de Ciencias de la Salud. Jaén. España
}

Qué se conoce: El cuidado familiar lo realizan fundamentalmente mujeres. Si no se comparte, genera una sobrecarga que altera el descanso y limita el desarrollo de otras actividades. Las expectativas de las cuidadoras varían en función de los problemas de salud. Qué aporta: Se muestran satisfechas con la competencia técnica y la comunicación de las enfermeras Demandan visitas domiciliarias más frecuentes. Educación sanitaria en técnicas específicas y en actividades de prevención y promoción de salud.

Trabajo financiado por el Fondo de Investigación Sanitaria, Ministerio de Sanidad y Consumo, expediente 98/0653, y Servicio Andaluz de Salud, expediente 97/23.

\section{Correspondencia:}

E. Gil.

Escuela Universitaria de Enfermería Universidad de Jaén. Paraje Lagunillas. Edificio B-3. 23071 Jaén. España.

Correo electrónico: egi@@ujaen.es

Aceptado para su publicación el 17-03-2005.

\section{Resumen}

Objetivos. Conocer la opinión y vivencias de las cuidadoras, y las expectativas y demandas con respecto a los profesionales de enfermería que realizan las visitas domiciliarias.

Método. Estudio cualitativo realizado en Andalucía en el año 1999. Participantes: mujeres cuidadoras de pacientes de cáncer en fase terminal, ancianos/as con demencia y enfermos terminales de sida. Estrategias para obtener información: entrevistas en profundidad y grupos de discusión. Análisis de contenido utilizando como marco de referencia el modelo de Virginia Henderson. Resultados. En los discursos de las cuidadoras se identificaron 6 tipos de cuidados realizados por las cuidadoras familiares: higiene, alimentación, eliminación, movilidad, realización de curas y administración de medicación. Las cuidadoras manifestaron su satisfacción con la competencia técnica y la comunicación de las enfermeras. Sin embargo, la valoración es deficiente con respecto a la frecuencia de las visitas domiciliarias y a la formación que reciben. Demandaron actividades formativas en técnicas específicas (curas, alimentación) y en actividades de prevención y promoción de salud (cambios posturales, ejercicios de movilidad).

Conclusiones. La labor realizada por las cuidadoras supone una sobrecarga emocional y de trabajo que afrontan, en muchos casos, sin tener la competencia técnica adecuada. Para mejorar las visitas domiciliarias de enfermería se debe aumentar la frecuencia de éstas y la formación de las cuidadoras.

Palabras clave: Atención domiciliaria. Enfermería. Necesidades de cuidados. Metodología cualitativa. Cuidadores familiares.

Gil-García E, Escudero-Carretero M, Prieto-Rodríguez MA, Frías-Osuna A. Vivencias, expectativas y demandas de cuidadoras informales de pacientes en procesos de enfermedad de larga duración. Enferm Clin. 2005;15(4):220-6.

\section{Abstract}

\section{Experiences, expectations and perceived needs of informal caregivers of patients with longstanding diseases}

Objectives. To determine informal caregivers' opinions and experiences of providing care and their expectations and needs concerning professional nurses who make home visits.

Method. A qualitative study was carried out in 1999 in the Autonomous Community of Andalusia. Participants: Female homecarers of terminally-ill patients with cancer or AIDS and elderly persons with dementia. Strategies for obtaining information: indepth interviews and discussion groups. The content analysis used Virginia Henderson's model as a framework.

Results. In the caregivers' statements, six types of care that they carried out related to hygiene, food, excretion, mobility, wound care, and medicine administration were identified. The caregivers were satisfied with professional nurses' technical and communication skills. However, their satisfaction with the number of visits and their own training was low. They requested training courses in specific skills (wound care, diet) and in prevention and health (postural changes, mobility exercises). Conclusions. The tasks carried out by the caregivers are physically and emotionally demanding, and caregivers frequently lack the required technical skills. To improve home care services, visits should be more frequent and caregivers' training should be up-graded.

Key words: Home care. Nursing. Care needs. Qualitative methodology. Nonprofessional carers. 


\section{Introducción}

La atención domiciliaria es una alternativa asistencial que proporciona cuidados integrales a personas que por su situación de salud o por problemas sociosanitarios requieren cuidados en el domicilio de carácter temporal o permanente ${ }^{1-4}$. Supone intervenciones como ayuda para el autocuidado, actividades de prevención y promoción de salud, cuidados psicosociales, actividades de refuerzo y apoyo a la red familiar y educación sanitaria ${ }^{2,5}$. Se dirige a pacientes que tienen dificultades para acceder al sistema sanitario público por diferentes motivos: encontrarse en fase terminal del proceso patológico, o tener limitaciones en la movilidad. Los programas de atención domiciliaria están cada vez más extendidos en la atención primaria debido a las características demográficas de la población y a los avances médicos y tecnológicos. Es previsible que se produzca un auge en su utilización y se conviertan en una alternativa de futuro ${ }^{4}$.

Uno de los objetivos de la atención domiciliaria es garantizar que el/la paciente reciba asistencia sanitaria con un nivel de efectividad similar al que obtendría en régimen de institucionalización ${ }^{5,6}$. Para ello, se debe contar con la aceptación del paciente, la familia y su colaboración. Un/a paciente en régimen de atención domiciliaria requiere vigilancia y atención permanentes y una intensa provisión de cuidados.

Dentro de la red familiar, el reparto de las cargas recae en una persona que asume la función de cuidador/a familiar ${ }^{7}$. El/la paciente establece con ella una relación basada en el cariño y el cuidado. Este tipo de relación es desempeñada fundamentalmente por mujeres $^{8}$. El desempeño de papel de cuidadoras durante largos períodos de tiempo puede ocasionar importantes secuelas físicas y emocionales derivadas de la sobrecarga de trabajo, del aislamiento y de la tensión emocional ${ }^{9}$. M. Ángeles Durán estima que el 88\% del total de cuidados que requiere un paciente de larga duración corre a cargo de esta red informal ${ }^{10}$. De hecho, se puede ir más lejos y afirmar que sin la colaboración y dedicación de estas mujeres, cuidadoras principales, los programas de atención domiciliara serían inviables. La implementación de la atención domiciliaria y la planificación de los trabajos enfermeros requieren conocer la opinión de las cuidadoras familiares, las pautas de cuidados que realizan y sus expectativas ${ }^{3}$.

La investigación que se presenta aquí tiene un doble objetivo: conocer la opinión y las vivencias emocionales de las cuidadoras e indagar en las expectativas y demandas que tienen con respecto a los profesionales de enfermería. Utilizamos como marco teórico el modelo de necesidades de cuidados de Henderson ${ }^{11}$.

\section{Método}

Investigación realizada en Andalucía durante el año 1999. Se entrevistó a personas que ejercían el papel de cuidadoras principales en 3 procesos de enfermedad: cáncer en fase terminal, ancianos con demencia y enfermos/as terminales de sida. La elección del diseño se debe al objeto de la investigación, que busca conocer y comprender opiniones y vivencias en el contexto social en el que se producen. La elección de los 3 procesos de enfermedad se debe a que son de larga duración y requieren cuidados de forma continuada.

\section{Muestreo}

Teniendo en cuenta la experiencia del equipo, la información aportada por profesionales de enfermería de atención domiciliaria y la bibliografía, como criterios de homogeneidad de los grupos se establecieron el sexo de la cuidadora, la patología del paciente (cáncer en fase terminal y pacientes con Alzheimer) y hábitat de residencia. Se buscó obtener discursos homogéneos y experiencias comunes. Pero como una excesiva homogeneidad inhibe al grupo, según Ibáñez, se decidió potenciar las diferencias entre las participantes y representar en el mismo grupo la estructura social, y se buscó la heterogeneidad en el grupo teniendo en cuenta la edad de las cuidadoras, el nivel de estudios y si recibían o no ayuda en el cuidado (tabla 1). Con estos criterios se pretendió que las personas tuvieran experiencias comunes pero, a la vez, se buscaba obtener riqueza en los discursos.

\section{Técnicas de recogida de información}

Se basaron en 8 grupos de discusión y en 8 entrevistas en profundidad. Los grupos se formaron con cuidadoras de enfermos de cáncer en situación terminal y cuidadoras de ancianos con demencia. En el caso de los pacientes con sida se recurrió a la técnica de la entrevista, a fin de mantener la confidencialidad y el anonimato de las personas que tienen la enfermedad (tabla 1). La moderación de todos los grupos y las entrevistas corrieron a cargo de la misma investigadora. En todos los casos se partía de un guión (tabla 2).

Las reuniones de grupos se realizaron en lugares conocidos por las cuidadoras pero asociados al ámbi- 


\section{TABLA 1. Criterios de selección de personas componentes de la muestra}

Criterios de homogeneidad en los grupos

Enfermedad del paciente, hábitat de residencia y sexo de la cuidadora

Criterios de heterogeneidad intragrupo

Edad: 3 categorías ( $<40,41-65$ y $>66$ años)

Nivel de estudios: bajo (analfabetos y estudios primarios),

medio (estudios medios) y superior (universitarios)

Ayuda en el cuidado: si recibe ayuda o no para el cuidado del paciente

Realización o no de un trabajo fuera del ámbito doméstico

Distribución de los grupos de discusión y entrevistas

4 grupos de discusión de cuidadoras de enfermos de cáncer en proceso terminal

2 grupos de cuidadoras que viven en ciudades de más de 100.000 habitantes:

G1 (9 mujeres), G2 (8 mujeres)

2 grupos de cuidadoras que viven en ciudades de menos de 100.000 habitantes: G3 (5 mujeres), G4 (3 mujeres)

4 grupos de discusión de cuidadoras de ancianos con demencia

2 grupos de cuidadoras que viven en ciudades de más de 100.000 habitantes:

G5 (8 mujeres), G6 (8 mujeres)

2 grupos de cuidadoras que viven en ciudades de menos de 100.000 habitantes: G7 (6 mujeres), G8 (3 mujeres)

8 entrevistas a cuidadoras de pacientes con sida en fase terminal

\section{TABLA 2. Guión de entrevistas y grupos}

Me gustaría que habláramos desde vuestra experiencia y me contárais ¿qué hacéis para cuidar a vuestro familiar? ¿Cómo lo hacéis?

Por favor, recordad el momento en el que le diagnosticaron la enfermedad a vuestro familiar. Contadme, por favor, ¿qué esperábais de los servicios sanitarios? ¿Qué encontrásteis?

Ahora me gustaría que hablásemos sobre el trabajo de las enfermeras. ¿Cómo lo veis? ¿En qué sentido? ¿Por qué ocurre?

Recordad un momento en que se haya presentado un problema en el proceso de la enfermedad de vuestro familiar. ¿Qué pasó, qué habéis hecho para solucionarlo? ¿Por qué habéis elegido esa estrategia?

¿Qué sensación tenéis cuando las enfermeras se van de vuestra casa?

to de la salud (asociaciones de vecinos y otros lugares de encuentro de la comunidad, como asociaciones de mujeres y centros de juventud). El tiempo de duración de cada reunión fue, aproximadamente, de $2 \mathrm{~h}$. Todas transcurrieron en un clima de cordialidad y satisfacción. Las entrevistas se realizaron en los domicilios procurando la máxima intimidad, con una duración aproximada de 1,5 h. La información que se obtuvo fue grabada con el consentimiento de los participantes y transcrita literalmente por profesionales.

\section{Captación y selección de participantes}

La selección de las participantes se llevó a cabo en 2 momentos: en primer lugar, se obtuvo un listado de las zonas básicas de salud en Andalucía y, mediante un muestreo aleatorio, se eligieron 4 centros de salud situados en ciudades de menos de 100.000 habitantes y otros 4 centros de salud situados en ciudades de más de 100.000 habitantes. Se contactó con profesionales de enfermería de los centros elegidos, que localizaron cuidadoras que cumplían los requisitos de los perfiles establecidos. Se acordó la cita por teléfono y se les envió una carta institucional. Días antes se confirmaba la participación con un recordatorio telefónico. Los/as investigadores/as elaboraron un contrato comunicativo en el que se establecía la información que debía aportarse con el objetivo de que todas las participantes obtuvieran la misma información.

\section{Análisis de la información}

El primer nivel de categorías básicas era:

- Percepción de las cuidadoras.

- Necesidades de las cuidadoras.

- Actividades de cuidados que realizaban.

Para valorar los cuidados se utilizó el modelo de necesidades de Virginia Henderson, que establece 14 necesidades humanas básicas: respirar, comer, beber, eliminar los desechos del organismo, descansar y dormir, seleccionar la vestimenta, mantener la temperatura corporal, mantener la higiene corporal, evitar los peligros del entorno, comunicarse, expresar emociones y ejercer el culto de acuerdo con las propias creencias. Posteriormente, las categorías se reagruparon (tabla 3 ).

En el proceso de análisis se asignaron fragmentos de texto a las categorías. Posteriormente se trianguló la codificación: 3 miembros del equipo codificaron la información buscando el consenso en los párrafos que presentaban codificaciones distintas. El apoyo del software NUDIST resultó de gran utilidad.

\section{Resultados}

\section{Vivencias de las cuidadoras sobre los cuidados que realizan}

Las cuidadoras consideraban que los cuidados que han de dispensar requieren una absoluta dedicación: "Mucho, desde acostarlo, a lavarlo, a darle de comer, a darle 
un cariño, a que tenga confianza, pues todo, si es que es todo el día" (G6). Se ocupaban de atender necesidades funcionales y de adaptación, pero además realizaban otras actividades que requieren competencia técnica, como la administración de medicación o la realización de curas, tareas que, en otros momentos asistenciales, son asumidas por profesionales del sistema sanitario público.

En los discursos se distinguieron distintas necesidades que ellas satisfacían: relacionadas con la alimentación, la higiene, la liminación, la movilidad, la realización de curas y la administración de medicación (tabla 4).

Las actividades relacionadas con la alimentación e higiene son las que menos esfuerzos les generaban: "Estoy muy volcada con él siempre... mucho cariño, de no darle ningún sinsabor, muy bien atendido en sus comidas, la limpieza, al máximo, eso que necesita también para el caso de mi marido, es muchísima, muchísima higiene, y estoy muy pendiente de él" (G1). Sin embargo, realizar otras tareas que requieren mayor competencia técnica, como aspiración de secreciones, control de la medicación o administración de medicación por vía intramuscular, les generaba inseguridad, ansiedad y temor. "Tampoco se puede pasar una con la medicación, se descompensa, se puede deshidratar, hay que controlársela porque se le baja el sodio, se intoxica, es peligroso, hay que jugar con un margen siempre, es muy peligroso" (G6); "Como está tomando mucha medicación, esto es insoportable. Se olvida... se tiene que tomar una pastilla a la hora de los aerosoles, jarabe, le tengo que poner una inyección, luego otro jarabe y la primera de todas hacer el control del azúcar por la mañana, en ayunas, al tomarse la leche" (G7). "Que el médico de cabecera, le manda las inyecciones... y él mismo le da la receta para que allí en la sala de curas le den las inyecciones y las agujas, él sabe que esta mujer se tiene que pinchar... Lo que no puede ser es que yo se las ponga, que vamos, que yo sola me he enseñado, pero lo mismo se las puedo poner un día bien, iy si se la pongo mal?, yo tampoco soy enfermera, que yo no sé" (E). "Al principio venía una ATS a ponerle la sonda (nasogástrica), pero claro, yo no voy a estar todos los días, que se le ha salido, que si esto, entonces, pues, he tenido que aprender yo sola y no me gusta pero se las pongo yo sola" (G7).

Es importante resaltar el hecho de que la mayoría de ellas no recibía ayuda para llevar a cabo los cuidados que realizaban y sentían una profunda soledad: "Como me ha pasado a mí, que me tengo que ir al médico y no puedo ir porque no tengo con quien dejarlo" (G2).
TABLA 3. Categorías de análisis

Percepción
Referencias sobre las experiencias y vivencias de los cuidados
Valoración
Referencias acerca de los cuidados que realizan los profesionales de enfermería
en las visitas domiciliarias
Necesidades
Recursos que las cuidadoras requieren para realizar mejor la labor de cuidados
Competencia técnica
Comunicación
Capacidad de respuesta
Formación
Actividades de cuidados
Referencias en los discursos sobre los cuidados que las cuidadoras informales
realizan
Higiene
Alimentación
Eliminación
Movilidad
Curas
Medicación

Sus vidas giraban en torno a los ritmos de cuidados establecidos para el paciente, el horario de comidas, la medicación, la movilización y el resto de los cuidados.

En los discursos se percibía un proceso de abandono de sí mismas y de sus necesidades, paralelo al proceso de enfermedad. Para muchas cuidar era una obligación moral que les generaba aislamiento social y les producía abatimiento, tristeza e inestabilidad emocional: "Hay días que lloras, hay días que ríes, tienes días de todo. Y otras veces que tengo mucha pena y es nada más que llorar" (G4). Se percibían a sí mismas como "encerradas" a la vez que realizaban una labor delicada y exigente. En muchos casos sentían confusión ante la incertidumbre del proceso de enfermedad y desconocían los apoyos con los que podían contar. El modo de afrontar el proceso y de asumir el rol depende de múltiples factores: contar con otros apoyos familiares o profesionales o haber recibido suficiente información y formación.

\section{Expectativas y demandas de las cuidadoras familiares}

En general, existe satisfacción y una buena valoración de la competencia técnica demostrada por las/os profesionales de enfermería que acuden al domicilio, basada en la percepción de que aplican adecuadamente las técnicas necesarias, como tomar la tensión, poner inyectables, realizar análisis de glucemias, curar y cam- 


\section{TABLA 4. Cuidados que realizaban las cuidadoras en sus domicilios}

Cuidados relacionados con la higiene

Todas las cuidadoras dedican mucha atención y esfuerzo a estas tareas porque las consideran importantes para prevenir complicaciones. La higiene del paciente es la primera actividad de cuidado que suelen efectuar cada día y es la actividad para la que suelen recibir más ayuda, incluso contratando a otras personas cuando las posibilidades de la familia lo permiten

Cuidados relacionados con la alimentación

Abarca distintos aspectos, desde preparar la comida cuidando que la dieta contenga la composición y nutrientes adecuados, hasta contemplar una forma de presentación acorde con el proceso de la enfermedad (trituración) y darles de comer cuando es necesario. Los cuidados relativos a la alimentación se complican cuando el paciente necesita alimentación por sonda nasogástrica, por la inseguridad que su manejo genera a las cuidadoras

Cuidados relacionados con la eliminación

Muchas cuidadoras manifiestan preocupación por esta necesidad, especialmente en el caso de pacientes inmovilizados/as. Se suelen ayudar de pañales y laxantes. A veces es necesario realizar la extracción manual de las heces, tarea que ha de enseñarles a realizar un/a profesional

Cuidados relacionados con la movilidad

Todas las cuidadoras entrevistadas son conscientes de la necesidad de movilizar al enfermo/a para evitar la formación de escaras y los riesgos y complicaciones de éstas, aunque la mayoría afirma que nadie les ha informado al respecto

Cuidados relacionados con la realización de curas Suelen realizarlas las cuidadoras principales cuando son pequeñas úlceras que requieren limpieza y antiséptico. En el caso de que se produzcan heridas mayores, les genera mucha ansiedad tener que atenderlas, y prefieren la intervención de un profesional

Administración de medicación

Las cuidadoras administran medicación, sobre todo analgésica, se encargan de su control, de su periodicidad y de valorar sus efectos y de vigilar posibles reacciones e, incluso, deciden sobre el tratamiento más oportuno. En ocasiones han de tomar decisiones importantes con respecto a la cantidad y la periodización de la dosis requerida

biar la sonda nasogástrica y vesical: "Estamos contentos con lo que nos hacen, respecto a como lo hacen y todo eso, sí, son profesionales y lo hacen bien" (G5). No obstante, consideraban que la competencia no sólo comprende aspectos técnicos realizados con precisión, fiabilidad y seguridad, sino que también incluye otros aspectos relacionados con habilidades de comunicación, como amabilidad e interés: "Pues, mira, lo mira, lo ausculta, le toma la tensión, muchas veces, la última vez le hizo lo del azúcar, le habla, nos dice a nosotros: ¿me entiendes?" (G2).

Las cuidadoras asociaban establecer una buena comunicación con sentirse escuchadas y valoradas en el desempeño del su función. Necesitaban que se reconociera su trabajo, encontrar alguien con quien poder compartir sus experiencias, sentirse apoyadas y reforzadas en sus decisiones, sentirse partícipes y recibir ánimos. Una de las principales funciones del personal de enfermería en la atención domiciliaria es proporcionar atención emocional y psicológica tanto al paciente como a la cuidadora: "Agradable, se pone a hablar con la abuela, después, se pone a hablar conmigo: mira Juanita, hay que ver lo bien que llevas esto y me explicaba las cosas y te parecía que encontrabas un consuelo" (G6). Dos de las cualidades esenciales del personal de enfermería a domicilio han de ser la capacidad de escucha y la capacidad de hacer partícipes del proceso asistencial a las cuidadoras. La cercanía, la amabilidad, el respeto y la consideración son aspectos imprescindibles para alguien que entra a trabajar a un domicilio privado. Asimismo, son esenciales la habilidad de empatizar, mostrar interés y tener capacidad para transmitir ánimos y consuelo: "Muy cariñosa con mi madre cuando va y te consuela, es muy dulce de verdad" (G6).

En los discursos aparece una valoración deficiente del profesional de enfermería como consecuencia de un excesivo distanciamiento de las visitas domiciliarias. Las cuidadoras destacaban la tardanza en la respuesta y el hecho de que las visitas fuesen escasas y sólo a demanda: "Nada, no vienen, tengo yo que llamarlos, ellos no son para decir: a ver este hombre si hay que cambiarle la sonda que muchas veces se le atranca o a ver si tiene algo inflamado" (G7). Les preocupaba especialmente que, ante una complicación, encontrasen una respuesta pronta y eficaz. Esta preocupación se expresaba con mayor fuerza en el ámbito rural, donde la accesibilidad a los servicios sanitarios era considerada más deficiente. Asimismo, consideraban que existe un vacío en el desarrollo de actividades formativas a los/as cuidadores/as, tanto en relación con el entrenamiento y formación de técnicas específicas como en el entrenamiento y formación de actividades de prevención y promoción de la salud (cambios posturales y ejercicios de movilidad). En general, las cuidadoras entrevistadas consideraban que los servicios públicos no les prestan la ayuda suficiente para proporcionar a los/as pacientes todos los cuidados que requieren.

\section{Discusión}

La metodología para realizar el estudio ha resultado muy adecuada, a la luz de los resultados, y ha permitido cumplir el objetivo ${ }^{13,14}$. Si bien el diseño metodológico cualitativo presenta como limitación que las 
conclusiones no pueden ser extrapoladas, permite profundizar en los discursos y señalar las líneas por las que debe transcurrir la mejora de los cuidados enfermeros. Como señala Carmen de la Cuesta ${ }^{15}$, la investigación cualitativa se convierte en una herramienta crucial al servicio de la profesión, ya que permite obtener un conocimiento individualizado y profundo, de primera mano, que posibilita la planificación de las acciones y las prácticas enfermeras para la atención integral y personalizada. La construcción de criterios de homogeneidad y heterogeneidad de los grupos permitió mantener simetría en la información y los discursos fueron productivos, a la vez que apreciamos las diferencias que todo discurso social posee.

Respecto a los resultados, hay varios aspectos que deben resaltarse. Los procesos de larga duración atendidos en el domicilio pueden ocasionar efectos adversos sobre la unidad familiar, en su organización y en la calidad de vida de sus miembros. Estas consecuencias se concentran en las personas que ejercen el rol de cuidadoras principales, quienes muestran una absoluta dedicación ${ }^{16}$, asumen a costa de su tiempo y esfuerzo la tarea de vigilar y atenderlos, incluso, a veces, descuidando su propia salud. Las características y condiciones de cuidar implican factores de impacto en la salud de las personas que ejercen mayoritariamente la tarea informal de cuidar: las mujeres ${ }^{17,18}$. Cuidar a personas dependientes exige una atención continua que, si no es una tarea compartida ${ }^{19}$, interfiere en la cantidad y calidad del descanso ${ }^{20}$. En general, ellas ven restringida su movilidad y las posibilidades de acudir a otras instituciones, entre ellas a los centros sanitarios para seguir tratamientos ${ }^{5}$.

El nivel de deterioro que el papel de cuidadora produce está asociado con la forma de afrontarlo. En su investigación, Artaso et $\mathrm{al}^{21}$ encontraron que el 79\% de las cuidadoras estaba sometido a una intensa sobrecarga y que las estrategias de afrontamiento más mencionadas y útiles fueron la búsqueda de apoyo social, la planificación, el autocontrol y la revaluación positiva. Afirman que si se incrementa en un punto el apoyo social, la sobrecarga desciende en un $12 \%$. El presente estudio, aunque con distinta metodología, revela asimismo que contar con apoyos y tener cierto control de la situación y del proceso son factores que ayudan al afrontamiento. En este sentido, se revela como esencial el apoyo del personal de enfermería en los programas domiciliarios, el apoyo emocional y la formación sobre los cuidados y técnicas como demandan las cuidadoras. La información sobre la evolución de la enfermedad y la forma de resolver los problemas que puedan ir surgiendo aumenta la sensación de seguridad y de control ${ }^{22-24}$.

Por otro lado, las expectativas que son un factor determinante en la satisfacción y que varían en función de los problemas de salud ${ }^{25}$ se deben constituir en ejes de actuación para mejorar y atender las necesidades de la población enferma o cuidadora ${ }^{26}$. Las cuidadoras demandan algo más que profesionales de enfermería técnicamente competentes. Las enfermeras deben ser técnicamente competentes, pero también deben desarrollar habilidades de relación, comunicación y fomentar actividades formativas. Se avecinan tiempos en los que las demandas de los/as pacientes y sus familiares ante los servicios sanitarios cambiarán ${ }^{27}$. Pero, ¿estamos preparados/as?

\section{Conclusiones}

Las mujeres entrevistadas perciben la tarea de cuidar como una sobrecarga de trabajo que afrontan con mucha dedicación y esfuerzo. La mayoría de las veces la asumen sin apoyo institucional y, en otras ocasiones, sin una red social y/o familiar suficiente. Esta situación genera una vivencia de abatimiento, aislamiento, tristeza e incluso de inestabilidad emocional. El modo de afrontar el proceso de enfermedad y de percibir la vivencia del cuidado está relacionado con los apoyos sociales y familiares.

Las intervenciones que las cuidadoras realizan con mayor frecuencia son actividades relacionadas con la alimentación, la higiene, la eliminación, la movilidad, la realización de curas y la administración de medicación. Algunas de estas actividades las llevan a cabo a pesar de que ellas consideran que no tienen una competencia técnica suficiente. Por último, encontramos una buena valoración de la competencia técnica y de las habilidades de comunicación de los profesionales de enfermería que acuden al domicilio, y una valoración deficiente con respecto a la accesibilidad del personal de enfermería, la frecuencia de visitas domiciliarias y la educación sanitaria que reciben.

\section{Bibliografía}

1. Benítez MA. Reflexiones sobre la realidad de la atención domiciliaria. Aten Primaria. 2003;31(8):478-9.

2. De la Revilla LA, Espinosa Almendro JM. La atención domiciliaria y la atención familiar en el abordaje de las enfermedades crónicas de los mayores. Aten Primaria. 2003;31(9):587-91. 
3. Minardi R, Morales M, Llopis A, Sette J, Ramón E, Días JA. La hospitalización domiciliaria: antecedentes, situación actual y perspectivas. Rev Panam Salud Pública. 2001;10(1):45-55.

4. Ruiz V, Valdivieso B. Hospitalización a domicilio y atención domiciliaria. Hacia la excelencia de los servicios. FMC-Formación Médica Continuada. Aten Primaria. 2001;8:537-42.

5. Comisión para el Desarrollo de la Atención Enfermera en el Servicio Andaluz de Salud (CODAE). Estrategias de mejora de la atención domiciliaria en Andalucía [serie en Internet] 2004 [citado 15 Nov 2003]. Disponible en: http://www.juntadeandalucia.es/servicioandaluzdesalud/principal/documentosAcc.asp?pagina=pr_desa_Innovacion1

6. Escarrabill J. La atención domiciliaria como alternativa a la hospitalización convencional. Aten Primaria. 2002;30(5):304-9.

7. García MM, Mateo I, Gutiérrez P. Cuidados y cuidadores en el sistema informal de salud. Investigación cuantitativa. Granada: EASP; 1999.

8. Domínguez C. Cuidado informal, redes de apoyo y políticas de vejez. Index Enferm. 1998;23:15-21.

9. Frías A, Pulido A. Cuidadores familiares de pacientes en el domicilio. Index Enferm. 2001;34:27-32

10. Durán MA. Los costes invisibles de la enfermedad. Bilbao: Fundación BBV; 2002.

11. Henderson V. La naturaleza de la enfermería: una definición y sus repercusiones en la práctica, la investigación y la educación. Madrid: McGraw-Hill; 1994.

12. Ibáñez J. Más allá de la sociología. El grupo de discusión: técnica y crítica. Madrid: Siglo XXI; 1986.

13. Hurley R. Qualitative research and the profund grasp of the obvious. Health Serv Res. 1999;34(5):1119-36.

14. Pope C, Mays N. Qualitative research: reaching the parts other methods cannot reach: an introduction to qualitative methods in health and health services research. BMJ. 1995;311:42-5.
15. Cuesta C. Contribución de la investigación a la práctica clínica de los cuidados de enfermería: la perspectiva de la investigación cualitativa. Enferm Clin. 1998;8(4):166-70.

16. De la Revilla L, Bailón E. La atención domiciliaria y la atención familiar en el anciano. Aten Primaria. 2003;10(4):249-54.

17. Bazo MT. El cuidado familiar en las personas ancianas con enfermedades crónicas: el caso de los pacientes con enfermedad de Alzheimer. Rev Gerontol. 1998;33(1):49-56.

18. Fernández I. Entre el dolor de espalda y la tristeza. La salud de las amas de casa de un barrio de Granada. Granada: Universidad; 1990.

19. Vallés MN. Problemas de salud y sociales de los cuidadores de los pacientes con demencia. Aten Primaria. 1998;22(8):481-5.

20. García MM, Fernández-Ruiz I, Mateo I. La salud de las mujeres en Andalucía. En: Instituto Andaluz de la Mujer. La situación social de las mujeres en Andalucía 1990-2000. Sevilla: Consejería de la Presidencia; 2001. p. 97-148.

21. Artaso B, Goño A, Biurrun A. Cuidados informales en la demencia: predicción de sobrecarga en cuidadoras familiares. Rev Gerontol. 2003;38(4):212-8

22. Richardson J. Health promotion in palliative care: the patients' perception of therapeutic interaction with the palliative nurse in the pri mare care setting. J Adv Nurs. 2002;40(4):432-40.

23. Rogero P. Atención domiciliaria. En: Frías A. Enfermería comunitaria. Barcelona: Masson; 2000. p. 217-30.

24. Ruiz MA, Muñoz MD, Maza MA, Martínez MN. Educación para la salud a cuidadores informales. Enferm Clin. 1998;8(4):171-7.

25. Prieto A, Gil García E, Heierle C, Frías A. La perspectiva de los cuidadores informales sobre la atención domiciliaria. Un estudio cualitativo con ayuda de un programa informático. Rev Esp Salud Pública. 2002;76(5):613-25.

26. Thompson A, Suñol R. Las expectativas como factores determinantes en la satisfacción de los pacientes: Conceptos, teoría y pruebas. Rev Calidad Asistencial. 1996;11:74-86.

27. Jadad A, Rizo CA, Enkin M. I am a good patient relieve it or not. BMJ. 2003;326(14):1293-4 